\title{
Pediatric melanoma: incidence, treatment, and prognosis
}

This article was published in the following Dove Press journal:

Pediatric Health, Medicine and Therapeutics

I8 April 2017

Number of times this article has been viewed

\author{
Faiez K Saiyed' \\ Emma C Hamilton' \\ Mary T Austin ${ }^{1,2}$ \\ 'Department of Pediatric Surgery, \\ McGovern Medical School, \\ ${ }^{2}$ Department of Surgical Oncology, \\ The University of Texas MD Anderson \\ Cancer Center, Houston, TX, USA
}

Correspondence: Mary T Austin

Department of Surgical Oncology, The

University of Texas MD Anderson

Cancer Center, I 400 Pressler, Unit I484,

Houston, TX 77030-1439, USA

$\mathrm{Tel}+\mathrm{I} 7137944408$

Fax + I 7137945720

Email MAustin@mdanderson.org

\begin{abstract}
The purpose of this review is to outline recent advancements in diagnosis, treatment, and prevention of pediatric melanoma. Despite the recent decline in incidence, it continues to be the deadliest form of skin cancer in children and adolescents. Pediatric melanoma presents differently from adult melanoma; thus, the traditional asymmetry, border irregularity, color variegation, diameter $>6 \mathrm{~mm}$, and evolution (ABCDE) criteria have been modified to include features unique to pediatric melanoma (amelanotic, bleeding/bump, color uniformity, de novo/any diameter, evolution of mole). Surgical and medical management of pediatric melanoma continues to derive guidelines from adult melanoma treatment. However, more drug trials are being conducted to determine the specific impact of drug combinations on pediatric patients. Alongside medical and surgical treatment, prevention is a central component of battling the incidence, as ultraviolet (UV)-related mutations play a central role in the vast majority of pediatric melanoma cases. Aggressive prevention measures targeting sun safety and tanning bed usage have shown positive sun-safety behavior trends, as well as the potential to decrease melanomas that manifest later in life. As research into the field of pediatric melanoma continues to expand, a prevention paradigm needs to continue on a community-wide level.
\end{abstract}

Keywords: melanoma, pediatric, adolescent, childhood

\section{Epidemiology}

Melanoma is the deadliest form of skin cancer and is the second leading cause of cancer in adolescents and young adults aged 15-29 years. ${ }^{1}$ The incidence of melanoma sharply increases with age from 1.1 per million in 1 - to 4 -year-olds to 10.4 per million in 15 - to 19 -year-olds. ${ }^{2}$ Since the 1970 s, the incidence of pediatric melanoma has increased with an average annual percent change of $2-2.9 \% .^{3-5} \mathrm{How}-$ ever, updated analyses of the Surveillance, Epidemiology, and End Results (SEER) database show decreasing overall trends from 2000 to $2010 .{ }^{6,7}$ Campbell et al ${ }^{7}$ found an $11.6 \%$ decrease in pediatric melanoma per year for children $<20$ years of age from 2004 to 2010. Within this demographic, higher rates of melanoma exist for children of older ages. ${ }^{8,9}$ There are also gender-based differences. Although females show a slightly higher incidence, both males and females show decreasing trends in melanoma incidence. ${ }^{7}$ Females older than 4 years have a higher incidence of lower body melanoma, and males older than 10 years have a higher incidence of head and neck melanoma. ${ }^{4}$ Furthermore, the majority of pediatric melanoma patients are non-Hispanic white. An analysis of SEER showed that $85 \%$ of melanoma cases in 
patients $<18$ years old were in non-Hispanic white patients, followed by Hispanic patients (5\%) and Asian/Pacific Islander patients $(2 \%){ }^{3}$

\section{Risk factors}

The majority of pediatric melanoma cases are sporadic and related to ultraviolet (UV) DNA damage..$^{9,10}$ UV light is associated with an increased risk of melanoma and changes in pigmentation..$^{5,11,12}$ This may have a more pronounced effect in older children and adults due to a cumulative UV exposure effect. Risk factors such as genetics, nevi, and family history can interact with UV exposure to cause melanoma in younger patients. ${ }^{9}$

Only $22 \%$ of pediatric melanoma patients have non-modifiable risk factors, including fair skin, xeroderma pigmentosum (XP), genetic susceptibility, pigmentation, age, nevi pattern, and family history. ${ }^{12} \mathrm{XP}$ is an autosomal recessive disorder associated with a defect in DNA excisional repair mechanisms, resulting in a 1000-fold increased risk of skin cancer. Associated skin malignancies include squamous cell carcinoma, basal cell carcinoma, and melanoma. Approximately 5\% of individuals with XP develop melanoma by a median age of 19 years. ${ }^{9}$ Familial melanoma accounts for $\sim 1 \%$ of cases of melanoma. ${ }^{13,14}$ Mutations in $C D K N 2 A$ are associated with $\sim 20-40 \%$ of families with three or more individuals with melanoma, while $C D K 4$ mutations have only been found in 17 families. Mutations in $C D K N 2 A$ and $C D K 4$ are associated with atypical nevi, earlyonset melanoma, and multiple primaries. ${ }^{13,14}$ Other genes associated with an increased susceptibility to melanoma among families and the general population include mutations in MITF, which confer a population-wide increased odds of melanoma of $1.7-4.8 .^{15,16}$

The presence of congenital nevi (CNM) is significantly correlated with melanoma. In a study of adolescents aged 15-19 years, the risk of melanoma was found to be 34 times higher in children with 100+ nevi and 15 times higher in children with $10+$ large nevi $>5 \mathrm{~mm}$ in diameter. ${ }^{9,17} \mathrm{~A}$ similar study conducted in Australia examined children younger than 15 years. In that study, Whiteman et al ${ }^{18}$ found multiple nevi and sun-sensitive characteristics, such as facial freckling and less tanning ability, to be indicative of a higher incidence of melanoma. Similarly, both inherited immunodeficiency and acquired immunosuppression, such as organ transplantation, are correlated with increased nevus density and higher rates of melanoma. ${ }^{9}$ Immunosuppressed patients with concerning nevi should be monitored and evaluated extensively, as these patients have an increased risk of melanoma.

\section{Pathology}

Pediatric melanoma can be subclassified into the following three main categories: conventional melanoma (CM), melanoma arising in CNM, and spitzoid melanoma. ${ }^{19}$ Differentiating between these subtypes is important because each type has different treatment options, risk factors, and histological findings. CMs show a high rate of single nucleotide variations (SNVs) that are characteristic of UV damage. ${ }^{20}$ This includes TERT-p mutations that result in a $\mathrm{C} \rightarrow \mathrm{T}$ coding change. As a result of this SNV, CM tumor cells have increased oncogene activity. ${ }^{20} \mathrm{CM}$ also shows genetic similarity to adult melanoma, suggesting the potential for further research on treatments based on adult melanoma protocols. ${ }^{19} \mathrm{CMs}$ and CNMs may be differentiated by $B R A F$ and NRAS mutations, respectively. $B R A F$ mutations in CMs only affect the mitogen-activated protein kinase pathway, thus requiring a supplementary PTEN mutation to result in melanoma. Since $N R A S$ functions in both the mitogen-activated protein kinase and the phosphatidylinositol 3 kinase/AKT pathway, an NRAS mutation in a CNM can lead to malignancy without a PTEN mutation. ${ }^{20}$ This is supported by evidence that melanoma arising in CNMs shows a lower frequency of UV-related mutations, possibly due to a higher baseline risk. ${ }^{19}$

There is a broad spectrum of atypical melanocytic neoplasms, and the distinction between Spitz nevus, atypical Spitz tumor, spitzoid melanoma, and melanocytic tumors of uncertain malignant potential is controversial with little diagnostic agreement among dermatopathologists. ${ }^{21-23}$ Many of these melanocytic lesions are misdiagnosed as benign and only recognized later to be malignant after they have recurred. In one study, $35 \%$ of the cases originally classified as spitzoid upon review were determined to be $\mathrm{CMs}$ with epithelioid or spindle cells. ${ }^{22}$ Because of the difficulty in differentiating benign from malignant melanocytic lesions in pediatric patients, all histological slides should be reviewed by dermatopathologists highly experienced in the diagnosis of such lesions.

Comparative genomic hybridization (CGH), fluorescence in situ hybridization (FISH), and immunohistochemistry (IHC) have been used as ancillary diagnostic tools to differentiate between melanoma and benign nevi in adults. ${ }^{24}$ Melanoma has a variety of patterns of chromosomal aberrations of multiple chromosomal gains and/or losses. Conversely, the majority of Spitz nevi patients have a normal karyotype. ${ }^{25}$ Atypical Spitz tumors represent a heterogeneous group with distinct genetic subtypes, including $B R A F V^{600 \mathrm{E}} / \mathrm{BAP} 1^{\text {neg }}$, HRAS mutant with increased copies of $11 \mathrm{p}$, and homozygous $9 \mathrm{p} 21$ deletion with negative p16 expression. ${ }^{25-27}$ Additionally, kinase fusions of $R O S 1, N T R K 1, A L K, B R A F$, and $R E T$ are found in the entire spectrum of spitzoid neoplasms in 
a mutually exclusive pattern. ${ }^{28}$ More recently, two 4-probe FISH assays targeting 6p25(RREB1), 11q13 (CCND1), 9p21 $(C D K N 2 A)$, and 8q24(C-MYC) and 6p25 (RREB1), 6q23 $(M Y B)$, Cep6 (centromere 6), and 11q13 (CCND1) used to differentiate between melanoma and benign nevi have been applied to Spitz tumors. ${ }^{22,29}$ However, caution must be exercised when evaluating an overall positive FISH assay as $20-23 \%$ of Spitz nevi are positive in at least one of the individual FISH probes. ${ }^{22,29}$ In conjunction with FISH and histological features of atypical Spitz tumors, the application of IHC panels assessing the expression of proteins involved in cell cycle regulation (Ki67, p16) and melanocytic markers (HMB45) can also be helpful in classifying spitzoid lesions. ${ }^{30,31}$

The clinical progression of spitzoid lesions is different from melanoma. Up to $39 \%$ of patients with atypical Spitz tumors will have nodal metastases. However, unlike patients with melanoma, nodal disease does not confer the same high mortality risk and there is rarely further disease progression. ${ }^{32-34}$ Several genetic markers have been identified as potential prognostic indicators. Among atypical Spitz tumors, gains in 6p25 (RREB1), 11q13 (CCND1), and homozygous deletions of 9p21 (CDKN2A) are associated with a higher risk of aggressive clinical behavior. TERT-p mutations, found in $>90 \%$ of CM, may also be a marker of more aggressive behavior when present in spitzoid lesions. ${ }^{20,35}$ Conversely, isolated 6q23 (MYB) loss and loss of 3p21 in BAP1-associated Spitz tumors are associated with a favorable clinical outcome..$^{27,29}$

\section{Presentation}

It is important to understand the different manifestations of pediatric melanoma as it varies across age groups. Previously, it was believed that younger children present with later stage lesions that are more likely to appear on the head and neck. ${ }^{3,36,37}$ Current research shows that melanoma location is relatively equally distributed across the body for children $<10$ years of age, although younger children of age $0-4$ years have a slightly lower incidence of truncal melanoma. Older children and adolescents aged 10-19 years have a higher incidence of truncal melanoma. ${ }^{7}$ Males also tend to present with lesions on the face and trunk, while females more often present with extremity tumors. . $^{12,36,38}$

\section{Diagnosis}

Early recognition is important to prevent progression of melanoma. Traditionally, the asymmetry, border irregularity, color variegation, diameter $>6 \mathrm{~mm}$, and evolution (ABCDE) criteria are used for clinical detection of melanoma. However,
Cordoro et al $^{10}$ found that $60 \%$ of children aged $<10$ years and $40 \%$ of adolescents did not meet the traditional ABCDE criteria. As a result, up to $82 \%$ of diagnoses took $>6$ months after detection and $62 \%$ of diagnoses took $>2$ months. ${ }^{10}$ Children presented with amelanosis, symmetry, regular borders, uniform color, and diameters of $\leq 6 \mathrm{~mm}$. Cordoro et $\mathrm{al}^{10}$ thus proposed the following criteria to encompass characteristics more specific to pediatric melanoma: $\mathrm{A}=$ amelanotic; $\mathrm{B}=$ bleeding, bump; $\mathrm{C}=$ color uniformity; $\mathrm{D}=$ de novo, any diameter; and $\mathrm{E}=$ evolution of mole..$^{10,37,39}$

A high index of suspicion is necessary when evaluating children. Dermatoscopic examination is important to visualize the morphologic features of pigmented and nonpigmented lesions to differentiate melanoma from nevi. ${ }^{34}$ Full-thickness biopsy is indicated for suspicious lesions. Excisional biopsies are recommended for concerning lesions. However, full-thickness punch biopsies are an acceptable alternative for specific locations (palms/soles, digits, face, or ears) or very large lesions. Shave biopsies can interfere with pathologic diagnosis and accurate measurements of Breslow thickness. Thus, shave biopsies should only be utilized for lesions with a very low index of suspicion. ${ }^{40}$

\section{Management}

The National Comprehensive Cancer Network (NCCN) outlined the surgical management of melanoma based on stages as follows: ${ }^{40}$

1. Stage $0-$ melanoma in situ or stage IA or IB with thickness $\leq 0.75 \mathrm{~mm}$, regardless of other features (ulceration or mitotic rate). Only a wide local excision (WLE) is warranted for these patients.

2. Stage IA - with a thickness $0.76-1.0 \mathrm{~mm}$ with no ulceration and a mitotic rate $0 / \mathrm{mm}^{2}$. For patients without adverse features (such as lesions $>0.75 \mathrm{~mm}$, positive deep margins, lymphovascular invasions, and invasion into reticular dermis), only WLE is recommended. Both WLE and sentinel lymph node biopsy (SLNB) are recommended if adverse features are present.

3. Stage IB - with thickness $0.76-1.0 \mathrm{~mm}$ with ulceration or a mitotic rate $\geq 1 / \mathrm{mm}^{2}$ or stage IB or II with thickness $>1.0 \mathrm{~mm}$, any feature (ulceration, any mitotic rate), and clinically negative nodes. Both WLE and SLNB are recommended for these patients.

4. Stage III - with clinically detected (palpable) positive nodes, microscopic satellites (from assessment of the primary lesion), and/or in-transit disease. For these patients, WLE and complete lymph node dissection (CLND) are warranted. CLND is recommended in cases with positive SLNB. 
5. Stage IV - distant metastases. Resection of the tumor and metastases is possibly indicated depending on variable factors, including the location of primary tumor/ metastases and progression of disease. ${ }^{41}$

SLNB is a minimally invasive staging tool used to determine if CLND and adjuvant therapy are warranted due to regional lymph node metasatases. ${ }^{42}$ In children, SLNB does not improve melanoma-specific survival, but it can be used as a prognostic indicator for poorer outcomes. ${ }^{43}$ In a study of 310 children $(<20$ years old $)$ with a melanoma of Breslow depth $>0.75 \mathrm{~mm}, 261$ underwent SLNB, with a melanomaspecific survival of $100 \%$ for the group with negative SLNB and $89 \%$ for the positive SLNB group. ${ }^{42}$

There are limited options for adjuvant therapy among pediatric patients with metastatic melanoma. Pediatric melanoma is a rare disease, and clinical trials for adults with metastatic melanoma have often excluded pediatric patients. Pegylated interferon alpha-2b (IFN $\alpha-2 b)$ is an adjuvant that has positively influenced disease-free survival in adults after melanoma resection. IFN $\alpha-2 b$ is also shown to be safe and feasible in children. ${ }^{44}$ Ipilimumab is a novel treatment for metastatic melanoma in adults, which was fast tracked for US Food and Drug Administration (FDA) approval after demonstrating improved survival in a pivotal Phase III clinical trial. ${ }^{45}$ Ipilimumab is a monoclonal antibody that binds to cytotoxic T-lymphocyte-associated antigen 4, blocking the downregulation of T-cell pathways that prevent autoimmunity. However, there are limited data on its efficacy in pediatric patients. While Phase I clinical trial among pediatric patients demonstrated safe toxicity levels, a Phase II clinical trial (NCT01696045) evaluating overall survival among pediatric patients with previously treated or untreated metastatic melanoma was terminated due to slow enrollment. ${ }^{46,47}$ An ongoing Phase II clinical trial (NCT02304458) is evaluating the efficacy of ipilimumab in combination with another monoclonal antibody, nivolumab. ${ }^{47}$ Vemurafenib is another promising agent for the treatment of melanoma with BRAF mutations. However, similar to ipilimumab, a Phase II clinical trial (NCT01519323) evaluating vemurafenib in pediatric patients with metastatic melanoma was terminated due to low enrollment.

Other ongoing trials include a vaccine study using the patient's own tumor cells to create a vaccine that was genetically modified to secrete GM-CSF to delay or stop the growth of the patient's tumor (NCT00258687), a Phase I/II study of pembrolizumab, and a Phase II study (NCT00539591) evaluating the combination of temozolomide with pegylated IFN $\alpha-2 b .{ }^{48-50}$ Further research is necessary to be able to determine effective combinations and protocols for these medications among pediatric patients with metastatic or recurrent melanoma.

\section{Outcomes}

Survival for pediatric melanoma has improved over the past 30 years, possibly due to improved staging. ${ }^{5}$ Five-year overall survival for all stages is $87-95 \% .^{4,5,38,43}$ Similar to adults, the main predictor of outcomes in melanoma is the stage at the time of diagnosis. ${ }^{4,5,38,43}$ Among patients with stage IV metastatic melanoma, the median survival time is $<1$ year and 5-year overall survival is $<12 \% .{ }^{4}$ Children $<10$ years old are more likely to present with a higher stage disease, which may in part be due to delayed diagnosis of children with melanoma. ${ }^{4,10,43}$

Data are conflicting regarding the prognostic role of age, gender, tumor thickness, ulceration, and sentinel lymph node status. In some studies, younger children $<10$ years old have significantly poorer survival as compared to adolescents, while in other studies, adolescents have decreased survival compared to younger children. ${ }^{4,9,43,51,52}$ The data are mixed regarding the contribution of patient gender to overall survival and the interaction with patient's age and overall survival. ${ }^{4,5,38,43,53}$ Younger children often present with thicker tumors than adults. ${ }^{4,7,36}$ However, the association between tumor thickness and survival may be different between adults and children., ${ }^{4,53}$ Conversely, other studies have demonstrated that increasing tumor thickness is associated with poorer survival among pediatric melanoma patients. ${ }^{5,38,43}$ Tumor ulceration is also more common among young children $<10$ years and is associated with worse outcomes. ${ }^{7,38,43}$ In addition, tumor thickness and ulceration are strong predictors of sentinel lymph node metastases among children. ${ }^{54}$ Similar to adults, a positive sentinel lymph node is associated with poorer prognosis. ${ }^{54,55}$ The prognostic implications of other traditional risk factors among adults with melanoma, including vertical growth phase, vascular invasion, and high mitotic activity, do not appear to be correlated with an increased risk of mortality. ${ }^{33,43,53}$

\section{Prevention}

UV light causes direct DNA mutations that are correlated with CM. ${ }^{5,12,56}$ The World Health Organization (WHO) has classified UV exposure as a class I carcinogen that is associated with a higher incidence of melanoma, especially in children with a higher exposure to sunlight and/or tanning beds. ${ }^{11,12,57} \mathrm{UV}$ exposure is a highly preventable cause of melanoma if sun-safety protocols are followed. The Surgeon General issued a notice about melanoma prevention and sun safety in 2014. This "Call to Action" is centered around five goals: increasing sun-safety behaviors, informing the public about risks and options, pushing policies for safe 
Table I Strategies to reduce UV exposure in children

\begin{tabular}{ll}
\hline Intervention & Example \\
\hline Tanning bed restrictions & a) State-level legislation limiting and banning tanning bed use among minors \\
Multilevel sun-safety counseling & a) In a cohort of 300 parents, I47 were given sun-safety counseling and I53 were provided a book, a swim shirt, \\
& and weekly text reminders about wearing sun screen \\
Community-wide sun-safety & a) SunWise program was implemented in elementary schools in Arizona to teach children about sun-safety \\
training & behaviors. \\
& b) SunSmart City program in New Jersey focused on working with parks, political advocacy, and small businesses \\
& to encourage sun-safety behaviors and education in the community
\end{tabular}

Abbreviation: UV, ultraviolet.

habits, reducing the harms of indoor tanning, and increasing monitoring and surveillance of outcomes..$^{58}$ Several strategies adopted to reduce UV exposure in children are listed in Table 1.

Indoor tanning confers a sixfold increased risk of developing melanoma among women younger than 30 years, and the first exposure typically starts in adolescents. ${ }^{59-61}$ Tanning bed-related lesions may manifest in late adolescence, but they continue to have a harmful impact throughout a patient's lifetime as UV exposure continues to accumulate. ${ }^{59,60,62}$ Public health efforts, including a black-box warning issued by the FDA and state-level legislation restricting their use by minors, have significantly decreased the use of indoor tanning among adolescents. ${ }^{63-65}$

Disseminating information of proper sun-protective behaviors (using sunscreen, staying in the shade, wearing wide brim hats, and wearing protective clothing) is important to prevent early acquisition of sunburn and UV-related DNA damage. Multicomponent interventions incorporating printed materials, interactive multimedia, and distribution of sunprotective products and community-wide and school-based prevention programs have been shown to increase adherence to clothing and sunscreen recommendations. ${ }^{66-68}$ For example, the SunWise Program developed by the Environmental Protection Agency to educate elementary and middle schoolaged children about sun-safety habits is estimated to have prevented $>11,000$ cases of skin cancer and 50 premature deaths by $2015 .{ }^{69}$

\section{Future directions}

Melanoma-specific survival for pediatric melanoma is increasing by $4 \%$ per year. ${ }^{5}$ Furthermore, recent studies have demonstrated that the rate of pediatric melanoma is decreasing. The overall prevalence and incidence are both relatively low and will continue to decrease if effective public health campaigns continue to be implemented. ${ }^{7}$ Future analyses of prevention techniques should begin to take into account cultural and sociological factors that affect adherence to sun-safety recommendations to ensure that preventions are not targeted solely at the fair-skinned individuals. For the Latino populations in the US, acculturation to US culture actually decreased sun-safety behaviors, partly related to a lack of perceived risk in darker-skinned populations. ${ }^{70}$ This underscores the importance of diverse interventions that target all demographics impacted by pediatric melanoma.

\section{Conclusion}

Pediatric melanoma has a growing body of knowledge, but more work is needed in implementing effective prevention and treatment. Pediatric melanoma is still poorly studied and underrepresented in clinical trials. ${ }^{71}$ Parallel studies between children and adult cohorts for surgical management and novel treatment options will allow standardized guidelines to be implemented. ${ }^{42}$ Simultaneously, more sun-safety protocols and recommendations can be created based on existing interventions that continue to show great potential in decreasing rates of melanoma.

\section{Disclosure}

There are no sources of outside financial support to disclose for any of the authors. The authors report no conflicts of interest in this work.

\section{References}

1. Barr RD, Ries LAG, Lewis DR, et al. Incidence and incidence trends of the most frequent cancers in adolescent and young adult Americans, including "nonmalignant/noninvasive" tumors. Cancer. 2016;122(7): 1000-1008.

2. U.S. Cancer Statistics Working Group. United States Cancer Statistics: 1999-2013 Incidence and Mortality Web-based Report. Atlanta: U.S. Department of Health and Human Services, Centers for Disease Control and Prevention and National Cancer Institute; 2016. Available from: www.cdc.gov/uscs. Accessed August 1, 2016.

3. Austin MT, Xing Y, Hayes-Jordan AA, Lally KP, Cormier JN. Melanoma incidence rises for children and adolescents: an epidemiologic review of pediatric melanoma in the United States. J Pediatr Surg. 2013;48(11): 2207-2213.

4. Lange JR, Palis BE, Chang DC, Soong S-J, Balch CM. Melanoma in children and teenagers: an analysis of patients from the National Cancer Data Base. J Clin Oncol. 2007;25(11):1363-1368.

5. Strouse JJ, Fears TR, Tucker MA, Wayne AS. Pediatric melanoma: risk factor and survival analysis of the surveillance, epidemiology and end results database. J Clin Oncol. 2005;23(21):4735-4741. 
6. Siegel DA, King J, Tai E, Buchanan N, Ajani UA, Li J. Cancer incidence rates and trends among children and adolescents in the United States, 2001-2009. Pediatrics. 2014;134(4):e945-e955.

7. Campbell LB, Kreicher KL, Gittleman HR, Strodtbeck K, Barnholtz-Sloan J, Bordeaux JS. Melanoma incidence in children and adolescents: decreasing trends in the United States. J Pediatr. 2015;166(6):1505-1513.

8. Tricoli JV, Blair DG, Anders CK, et al. Biologic and clinical characteristics of adolescent and young adult cancers: acute lymphoblastic leukemia, colorectal cancer, breast cancer, melanoma, and sarcoma. Cancer. 2016;122(7):1017-1028.

9. Pappo AS. Melanoma in children and adolescents. Eur J Cancer. 2003;39(18):2651-2661.

10. Cordoro KM, Gupta D, Frieden IJ, McCalmont T, Kashani-Sabet M. Pediatric melanoma: results of a large cohort study and proposal for modified ABCD detection criteria for children. J Am Acad Dermatol. 2013;68(6):913-925.

11. Sender L, Zabokrtsky KB. Adolescent and young adult patients with cancer: a milieu of unique features. Nat Rev Clin Oncol. 2015;12(8):465-480.

12. Wong JR, Harris JK, Rodriguez-Galindo C, Johnson KJ. Incidence of childhood and adolescent melanoma in the United States: 1973-2009. Pediatrics. 2013;131(5):846-854.

13. Aoude LG, Wadt KAW, Pritchard AL, Hayward NK. Genetics of familial melanoma: 20 years after CDKN2A. Pigment Cell Melanoma Res. 2015;28(2):148-160.

14. Kefford BRF, Bishop JAN, Bergman W. Counseling and DNA testing for individuals perceived to be genetically predisposed to melanoma: a consensus statement of the Melanoma Genetics Consortium. J Clin Oncol. 1999;17(10):3245-3251.

15. Bertolotto C, Lesueur F, Giuliano S, et al. A SUMOylation-defective MITF germline mutation predisposes to melanoma and renal carcinoma. Nature. 2011;480(7375):94-98.

16. Berwick M, Macarthur J, Orlow I, et al. MITF E318K's effect on melanoma risk independent of, but modified by, other risk factors. Pigment Cell Melanoma Res. 2014;27(3):485-488.

17. Youl P, Aitken J, Hayward N, et al. Melanoma in adolescents: a casecontrol study of risk factors in Queensland, Australia. Int $J$ Cancer. 2002;98(1):92-98.

18. Whiteman DC, Valery P, McWhirter W, Green AC. Risk factors for childhood melanoma in Queensland, Australia. Int $J$ Cancer. 1997;70(1):26-31.

19. Pappo AS. Pediatric melanoma: the whole (genome) story. Am Soc Clin Oncol Educ Book. 2014:e432-e435.

20. Lu C, Zhang J, Nagahawatte P, et al. The genomic landscape of childhood and adolescent melanoma. J Invest Dermatol. 2015;135(3):816-823.

21. Berk DR, Labuz E, Dadras SS, Johnson DL, Swetter SM. Melanoma and melanocytic tumors of uncertain malignant potential in children, adolescents and young adults - the Stanford experience 1995-2008. Pediatr Dermatol. 2010;27(3):244-254.

22. Massi D, Tomasini C, Senetta R, et al. Atypical Spitz tumors in patients younger than 18 years. J Am Acad Dermatol. 2015;72(1):37-46.

23. Zhao G, Lee KC, Peacock S, et al. The utilization of spitz-related nomenclature in the histological interpretation of cutaneous melanocytic lesions by practicing pathologists: results from the M-Path study. J Cutan Pathol. 2017;44(1):5-14.

24. Gerami P, Li G, Pouryazdanparast P, et al. A highly specific and discriminatory FISH assay for distinguishing between benign and malignant melanocytic neoplasms. Am J Surg Pathol. 2012;36(6):808-817.

25. Bastian BC, LeBoit PE, Pinkel D. Mutations and copy number increase of HRAS in Spitz nevi with distinctive histopathological features. $\mathrm{Am}$ J Pathol. 2000;157(3):967-972.

26. Wiesner T, Murali R, Fried I, et al. A distinct subset of atypical Spitz tumors is characterized by BRAF mutation and loss of BAP1 expression. Am J Surg Pathol. 2012;36(6):818-830.

27. Yazdan P, Cooper C, Sholl LM, et al. Comparative analysis of atypical spitz tumors with heterozygous versus homozygous $9 \mathrm{p} 21$ deletions for clinical outcomes, histomorphology, BRAF mutation, and p16 expression. Am J Surg Pathol. 2014;0(0):1-8.
28. Wiesner T, He J, Yelensky R, et al. Kinase fusions are frequent in Spitz tumors and Spitzoid melanomas. Nat Commun. 2014;5:3116.

29. Gerami P, Scolyer RA, Xu X, et al. Risk assessment for atypical spitzoid melanocytic neoplasms using FISH to identify chromosomal copy number aberrations. Am J Surg Pathol. 2013;37(5):676-684.

30. Puri PK, Ferringer TC, Tyler WB, Wilson ML, Kirchner HL, Elston DM. Statistical analysis of the concordance of immunohistochemical stains with the final diagnosis in spitzoid neoplasms. Am J Dermatopathol. 2011;33(1):72-77.

31. Cho-Vega JH. A diagnostic algorithm for atypical spitzoid tumor: guidelines for immunohistochemical and molecular assessment. Lab Investig. 2016;96(7):127A.

32. Dika E, Fanti PA, Fiorentino M, et al. Spitzoid tumors in children and adults: a comparative clinical, pathological, and cytogenetic analysis. Melanoma Res. 2015;25(4):295-301.

33. Stanelle EJ, Busam KJ, Rich BS, et al. Early-stage non-Spitzoid cutaneous melanoma in patients younger than 22 years of age at diagnosis: long-term follow-up and survival analysis. J Pediatr Surg. 2015;50(6):1019-1023.

34. Hawryluk EB, Liang MG. Pediatric melanoma, moles, and sun safety. Pediatr Clin North Am. 2014;61(2):279-291.

35. Davar D, Lin Y, Kirkwood JM. Unfolding the mutational landscape of human melanoma. J Invest Dermatol. 2015;135(3):659-662.

36. Aldrink JH, Selim MA, Diesen DL, et al. Pediatric melanoma: a single-institution experience of 150 patients. J Pediatr Surg. 2009;44(8):1514-1521.

37. Rajput A, Faizi SA, Nir I, et al. Pediatric melanoma in New Mexico American Indians, Hispanics, and non-Hispanic whites, 1981-2009. Am J Surg. 2014;207(3):412-416.

38. Brecht IB, Garbe C, Gefeller O, et al. 443 paediatric cases of malignant melanoma registered with the German Central Malignant Melanoma Registry between 1983 and 2011. Eur J Cancer. 2015;51(7): 861-868.

39. Ferrari A, Bisogno G, Cecchetto G, et al. Cutaneous melanoma in children and adolescents: the Italian rare tumors in pediatric age project experience. J Pediatr. 2014;164(2):376-382.e1-2.

40. NCCN Guidelines Version 3.2016 Panel Members Melanoma [webpage on the Internet]. NCCN Clinical Practice Guidelines in Oncology (NCCN Guidelines ${ }^{\circledR}$ ) Melanoma, Version 3.2016. 2016. Available from: https://www.nccn.org/professionals/physician_gls/f_guidelines. asp\#site. Accessed December 10, 2016.

41. Slade AD, Austin MT. Childhood melanoma: an increasingly important health problem in the USA. Curr Opin Pediatr. 2014;26(3):356-361.

42. Kim J, Sun Z, Gulack BC, et al. Sentinel lymph node biopsy is a prognostic measure in pediatric melanoma. J Pediatr Surg. 2016;51(6):986-990.

43. Averbook BJ, Lee SJ, Delman KA, et al. Pediatric melanoma: analysis of an international registry. Cancer. 2013;119(22):4012-4019.

44. Navid F, Herzog CE, Sandoval J, et al. Feasibility of pegylated interferon in children and young adults with resected high-risk melanoma. Pediatr Blood Cancer. 2016;63(7):1207-1213.

45. Hodi SF, O'Day SJ, McDermott DF, et al. Improved survival with ipilimumab inpatients with metastatic melanoma. $N$ Engl $J$ Med. 2010;363(8):711-723.

46. Merchant M, Wright M, Baird K, et al. Phase 1 clinical trial of ipilimumab in pediatric patients with advanced solid tumors. Clin Cancer Res. 2015;22(20):1364-1371.

47. National Cancer Institute [webpage on the Internet]. Nivolumab with or without Ipilimumab in Treating Younger Patients with Recurrent or Refractory Solid Tumors or Sarcomas. Available from: https://www. cancer.gov/about-cancer/treatment/clinical-trials/search/view?cdrid= $767035 \&$ version $=$ HealthProfessional\&protocolsearchid $=15753539$. Accessed February 20, 2017.

48. Clinicaltrials.gov [webpage on the Internet]. Vaccine Trial for Clear Cell Sarcoma, Pediatric Renal Cell Carcinoma, Alveolar Soft Part Sarcoma and Children with Stage IV Melanoma-Full Text View-ClinicalTrials. gov. Available from: https://clinicaltrials.gov/ct2/show/NCT00258687? term=melanoma+AND+pediatric\&rank=3. Accessed February 7, 2017. 
49. ClinicalTrials.gov [webpage on the Internet]. Phase II Study Incorporating Pegylated Interferon in the Treatment for Children with High-Risk Melanoma - Full Text View - ClinicalTrials.gov. Available from: https:// clinicaltrials.gov/ct2/show/NCT00539591?term=melanoma+AND+pe diatric\&rank=6. Accessed February 7, 2017.

50. Clinicaltrials.gov [webpage on the Internet]. A Study of Pembrolizumab (MK-3475) in Pediatric Participants with Advanced Melanoma or Advanced, Relapsed, or Refractory PD-L1-Positive Solid Tumors or Lymphoma (MK-3475-051/KEYNOTE-051) - Full Text View - ClinicalTrials.gov. Available from: https:/clinicaltrials.gov/ct2/show/NCT02 332668 term $=$ melanoma $+A N D+$ pediatric $\&$ rank $=4$. Accessed March 7, 2017.

51. Lewis KG. Trends in pediatric melanoma mortality in the United States, 1968 through 2004. Dermatol Surg. 2008;34(2):152-159.

52. Ferrari A, Bono A, Baldi M, et al. Does melanoma behave differently in younger children than in adults? A retrospective study of 33 cases of childhood melanoma from a single institution. Pediatrics. 2005; 115(3):649-654

53. Paradela S, Fonseca E, Pita-Fernández S, et al. Prognostic factors for melanoma in children and adolescents: a clinicopathologic, single-center study of 137 patients. Cancer. 2010;116(18):4334-4344.

54. Mu E, Lange JR, Strouse JJ. Comparison of the use and results of sentinel lymph node biopsy in children and young adults with melanoma. Cancer. 2012;118(10):2700-2707.

55. Moore-Olufemi S, Herzog C, Warneke C, et al. Outcomes in pediatric melanoma. Ann Surg. 2011;253(6):1211-1215.

56. Kirkwood J, Jukic D, Averbook B, Sender L. Melanoma in pediatric, adolescent and young adult patients. Semin Oncol. 2009;36(5):419-431.

57. El Ghissassi F, Baan R, Straif K, et al. A review of human carcinogens - part D: radiation. Lancet Oncol. 2009;10(8):751-752.

58. Gershenwald JE, Guy GP. Stemming the rising incidence of melanoma: calling prevention to action. J Natl Cancer Inst. 2016;108(1):2015-2017.

59. Lazovich D, Isaksson Vogel R, Weinstock MA, Nelson HH, Ahmed RL, Berwick M. Association between indoor tanning and melanoma in younger men and women. JAMA Dermatol. 2016;152(3):268-275.
60. Cust AE, Armstrong BK, Goumas C, et al. Sunbed use during adolescence and early adulthood is associated with increased risk of early-onset melanoma. Int J Cancer. 2011;128(10):2425-2435.

61. Guy GP, Berkowitz Z, Tai E, Holman DM, Everett Jones S, Richardson LC. Indoor tanning among high school students in the United States, 2009 and 2011. JAMA Dermatol. 2014;30341(5):1-11.

62. Boniol M, Autier P, Boyle P, Gandini S. Cutaneous melanoma attributable to sunbed use: systematic review and meta-analysis. $B M J$. 2012;345:e4757.

63. Gottlieb M, Balk SJ, Geller AC, Gershenwald JE. Teens and indoor tanning: time to act on the US food and drug administration's black-box warning. Ann Surg Oncol. 2014;22(3):701-703.

64. Skin Cancer Prevention Progress Report 2016. Atlanta, GA: Centers for Disease Control and Prevention, US Dept of Health and Human Services; 2016.

65. Centers for Disease Control and Prevention. Youth risk behavior surveillance — United States, 2015. Morb Mortal Wkly Rep. 2015;65(6):44-48.

66. Crane LA, Asdigian NL, Barón AE, et al. Mailed intervention to promote sun protection of children: a randomized controlled trial. Am J Prev Med. 2012;43(4):399-410.

67. Dobbinson SJ, Volkov A, Wakefield MA. Continued impact of sunsmart advertising on youth and adults' behaviors. Am J Prev Med. 2015;49(1):20-28.

68. Ho BK, Reidy K, Huerta I, et al. Effectiveness of a multicomponent sun protection for young children. JAMA Pediatr. 2016;170(4):334-342.

69. EPA [webpage on the Internet]. The History of the SunWise Program at EPA. 2016. Available from: https://www.epa.gov/sunsafety/historysunwise-program-epa. Accessed August 6, 2016.

70. Andreeva VA, Unger JB, Yaroch AL, Cockburn MG, BaezcondeGarbanati L, Reynolds KD. Acculturation and sun-safe behaviors among US Latinos: findings from the 2005 Health Information National Trends Survey. Am J Public Health. 2009;99(4):734-741.

71. Bleyer WA. Cancer in older adolescents and young adults: epidemiology, diagnosis, treatment, survival, and importance of clinical trials. Med Pediatr Oncol. 2002;38(1):1-10.
Pediatric Health, Medicine and Therapeutics

\section{Publish your work in this journal}

Pediatric Health, Medicine and Therapeutics is an international, peerreviewed, open access journal publishing original research, reports, editorials, reviews and commentaries. All aspects of health maintenance, preventative measures and disease treatment interventions are addressed within the journal. Practitioners from all disciplines are invited to submit

\section{Dovepress}

their work as well as healthcare researchers and patient support groups. The manuscript management system is completely online and includes a very quick and fair peer-review system. Visit http://www.dovepress.com/ testimonials.php to read real quotes from published authors. 